\title{
Derivation of seawater intrusion models by formal asymptotics
}

\author{
M. JAzAR ${ }^{*}$, R. Monneau ${ }^{a}$
}

March 4, 2014

\begin{abstract}
In this paper, we consider the flow of fresh and saltwater in a saturated porous medium in order to describe the seawater intrusion. Starting from a formulation with constant densities respectively of fresh and saltwater, whose velocities are proportional to the gradient of pressure (Darcy's law), we consider the formal asymptotic limit as the aspect ratio between the thickness and the horizontal length of the porous medium tends to zero. In the limit of the regime defined by the Dupuit-Forchheimer condition, we identify reduced models of Boussinesq type both in the cases of unconfined and confined aquifers.
\end{abstract}

MSC: $35 \mathrm{R} 35,35 \mathrm{~B} 40$

Keywords: seawater intrusion, formal asymptotics, porous medium, groundwater flow, Dupuit-Forchheimer, saltwater and freshwater interface, Ghyben-Herzberg relation, confined and unconfined aquifer, shallow water, Boussinesq equation.

\section{Introduction}

We are interested in the modeling of seawater intrusion in coastal regions. On the one hand coastal aquifers contain freshwater and on the other hand saltwater from the sea can enter the ground and replace the freshwater. This phenomenon can be especially important in coastal regions where there is intensive extraction of freshwater from wells. We refer to [7] for a general overview on seawater intrusion models.

Our main goal is to derive formally simplified (2D) models describing the evolution of the interfaces freshwater/saltwater and freshwater/dry soil, from common (3D) models of hydrology based on Darcy's law.

\subsection{Setting of the problem}

We consider two simple situations: the case of an unconfined aquifer (see Figure 1) and the case of a confined aquifer (see Figure 2). In each case, the constant seawater level is $\tilde{h}_{1}$ and the domains are assumed to be unbounded horizontally. The unboundedness of the domains does not create difficulties, because we restrict our approach to a formal level.

\subsubsection{The unconfined aquifer}

The geometry

We consider coordinates $(\tilde{x}, \tilde{z}) \in \mathbb{R}^{N} \times \mathbb{R}$ of the space with $\tilde{x}$ for the horizontal coordinate and $\tilde{z}$ for the vertical coordinate. In physical application, we have $N=1$ or $N=2$. We assume (see Figure 1 ) that the surface of the soil is described by the level $\tilde{z}=\tilde{a}(\tilde{x})$, while the interface of the impermeable bedrock

\footnotetext{
*LaMA-Liban, Lebanese University, P.O. Box 37 Tripoli, Lebanon. E-mail: mjazar@laser-lb.org

${ }^{a}$ Université Paris-Est, CERMICS, Ecole des Ponts ParisTech, 6 et 8 avenue Blaise Pascal, Cité Descartes Champs-sur-Marne, 77455 Marne-la-Vallée Cedex 2, France. E-mail: monneau@cermics.enpc.fr
} 


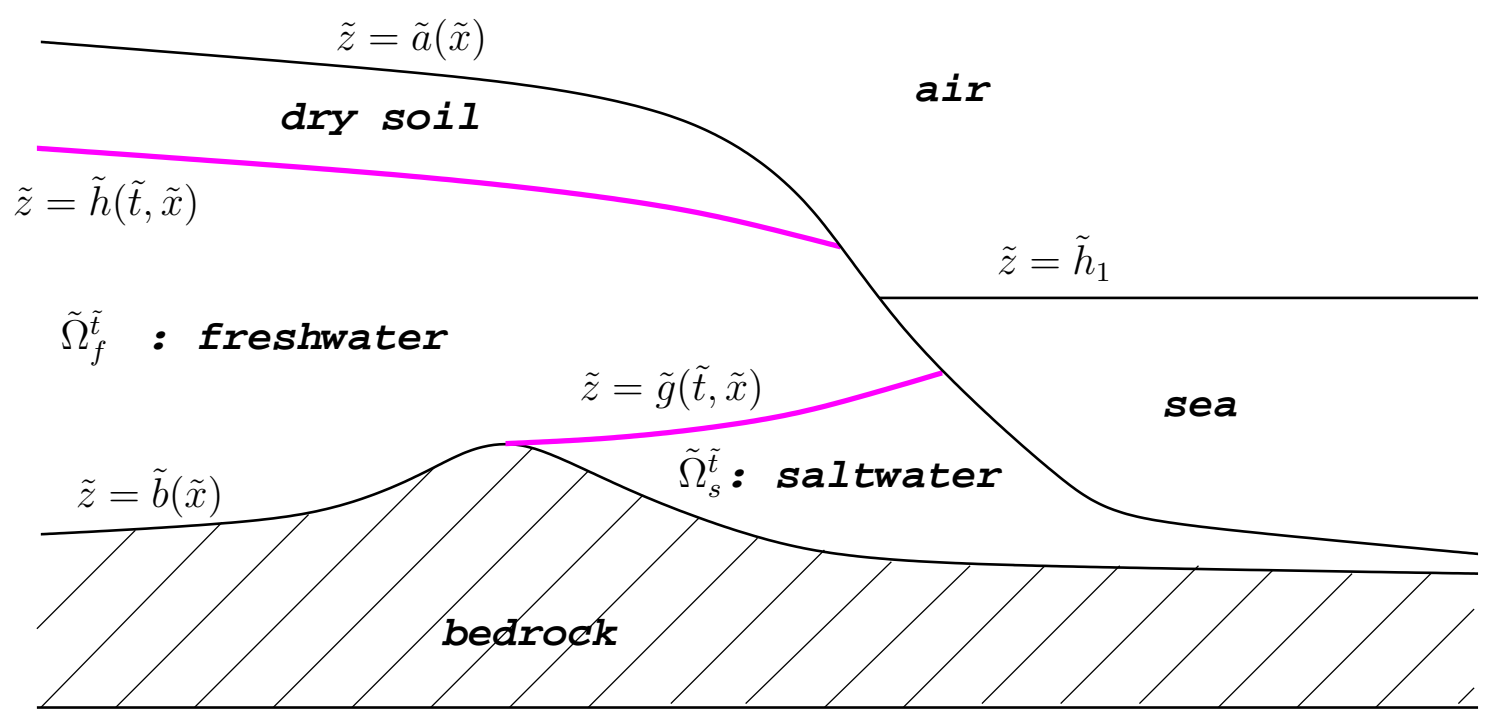

Figure 1: Unconfined aquifer

is described by the level $\tilde{z}=\tilde{b}(\tilde{x})$, satisfying $\tilde{b}<\tilde{a}$. We assume that in the porous medium, the interface between the freshwater and the dry soil can be written

$$
\Gamma_{\tilde{h}}^{\tilde{t}}=\left\{(\tilde{x}, \tilde{z}) \in \mathbb{R}^{N} \times \mathbb{R}, \quad \tilde{z}=\tilde{h}(\tilde{t}, \tilde{x})\right\},
$$

the interface between the saltwater and the freshwater (which are assumed immiscible) can be written

$$
\Gamma_{\tilde{g}}^{\tilde{t}}=\left\{(\tilde{x}, \tilde{z}) \in \mathbb{R}^{N} \times \mathbb{R}, \quad \tilde{z}=\tilde{g}(\tilde{t}, \tilde{x})\right\} .
$$

In particular these interfaces $\Gamma_{\tilde{h}}^{\tilde{t}}$ and $\Gamma_{\tilde{g}}^{\tilde{t}}$ are unbounded horizontally, and we have the following constraint

$$
\tilde{b} \leq \tilde{g} \leq \tilde{h} \leq \tilde{a} \quad \text { on } \quad \mathbb{R}^{N} .
$$

We assume that all the functions $\tilde{b}, \tilde{g}, \tilde{h}, \tilde{a}$ are smooth enough. We define the open set of freshwater

$$
\tilde{\Omega}_{f}^{\tilde{t}}=\left\{(\tilde{x}, \tilde{z}) \in \mathbb{R}^{N} \times \mathbb{R}, \quad \tilde{g}(\tilde{t}, \tilde{x})<\tilde{z}<\tilde{h}(\tilde{t}, \tilde{x})\right\} \quad \text { and } \quad \tilde{\Omega}_{f}=\bigcup_{\{\tilde{t}>0\}}\{\tilde{t}\} \times \tilde{\Omega}_{f}^{\tilde{t}}
$$

and the open set of saltwater in the porous medium

$$
\tilde{\Omega}_{s}^{\tilde{t}}=\left\{(\tilde{x}, \tilde{z}) \in \mathbb{R}^{N} \times \mathbb{R}, \quad \tilde{b}(\tilde{x})<\tilde{z}<\tilde{g}(\tilde{t}, \tilde{x})\right\} \quad \text { and } \quad \tilde{\Omega}_{s}=\bigcup_{\{\tilde{t}>0\}}\{\tilde{t}\} \times \tilde{\Omega}_{s}^{\tilde{t}} .
$$

Similarly, we define the open set of the porous medium as

$$
\tilde{\Omega}^{\tilde{t}}=\left\{(\tilde{x}, \tilde{z}) \in \mathbb{R}^{N} \times \mathbb{R}, \quad \tilde{z}<\tilde{a}(\tilde{x})\right\} \quad \text { and } \quad \tilde{\Omega}=\bigcup_{\{\tilde{t}>0\}}\{\tilde{t}\} \times \tilde{\Omega}^{\tilde{t}}
$$

where in order to keep uniform notations, we use the notation $\tilde{\Omega}^{\tilde{t}}$ even if it is independent of $\tilde{t}$.

The PDEs

We set $\alpha=f$ for the freshwater and $\alpha=s$ for the saltwater. We define the density field of the fluid $\alpha$ as

$$
\tilde{\rho}_{\alpha}(\tilde{t}, \tilde{x}, \tilde{z})= \begin{cases}\rho_{\alpha}^{0} & \text { if } \quad(\tilde{x}, \tilde{z}) \in \tilde{\Omega}_{\alpha}^{\tilde{t}} \\ 0 & \text { otherwise }\end{cases}
$$


where $\rho_{\alpha}^{0}$ is the mass density of the fluid $\alpha$ (assumed to be a constant with $0<\rho_{f}^{0}<\rho_{s}^{0}$ ). We also set the specific weight $\gamma_{\alpha}=\rho_{\alpha}^{0} g^{0}$ with $g^{0}$ the standard gravity constant. We assume that $\tilde{\rho}_{\alpha}$ solves formally the following equations

$$
\left\{\begin{array}{l}
\tilde{\phi}_{\alpha}(\tilde{x}, \tilde{z}) \frac{\partial \tilde{\rho}_{\alpha}}{\partial \tilde{t}}+\widetilde{\operatorname{div}}\left(\tilde{\rho}_{\alpha} \tilde{v}_{\alpha}\right)=0 \quad \text { on } \quad \tilde{\Omega}_{\alpha} \\
\tilde{v}_{\alpha}=-\tilde{\kappa}_{\alpha}(\tilde{x}, \tilde{z}) \widetilde{\nabla}\left(\tilde{p}+\gamma_{\alpha} \tilde{z}\right) \quad \text { on } \quad \tilde{\Omega}_{\alpha}
\end{array} \mid \begin{array}{l}
\text { for } \alpha=f, s \\
\tilde{p} \quad \text { is continuous across } \quad \Gamma_{\tilde{g}}^{\tilde{t}} \\
\tilde{v}_{f} \cdot \tilde{n} \geq 0 \quad \text { on } \quad\left(\partial \tilde{\Omega}^{\tilde{t}}\right) \cap\left(\partial \tilde{\Omega}_{f}^{\tilde{t}}\right) \\
\tilde{g} \leq \tilde{h}_{1} \text { and } \tilde{b}<\tilde{h} \quad \text { everywhere } \\
\tilde{\phi}_{\alpha} \frac{\partial \tilde{F}_{\alpha}}{\partial \tilde{t}}+\tilde{v}_{\alpha} \cdot \tilde{\nabla} \tilde{F}_{\alpha}=0 \quad \text { on }\left\{\tilde{F}_{\alpha}=0\right\} \cap \tilde{\Omega}_{\alpha} \cap \tilde{\Omega} \text { for } \tilde{F}_{\alpha}+\tilde{z}=\left\{\begin{array}{lll}
\tilde{h}, \tilde{g}, \tilde{b} & \text { if } \quad \alpha=f, \\
\tilde{g}, \tilde{b} & \text { if } \quad \alpha=s .
\end{array}\right.
\end{array}\right.
$$

Here $0<\tilde{\phi}_{\alpha}(\tilde{x}, \tilde{z}) \leq 1$ is the effective porosity of the porous medium, where, in order to simplify for a fully saturated medium, we assume that the water content is equal to the porosity. Notice that this effective porosity $\tilde{\phi}_{\alpha}$ should be independent of the fluid $\alpha$, but for sake of generality we allow here such a dependence.

Here $\tilde{p}$ is the pressure assumed to be defined on $\overline{\tilde{\Omega}_{f}^{\tilde{t}} \cup \tilde{\Omega}_{s}^{\tilde{t}}}$, and $\widetilde{\operatorname{div}}$ and $\widetilde{\nabla}$ are the divergence and the gradient, respectively, taken with respect to the coordinates $(\tilde{x}, \tilde{z})$. Moreover $\tilde{\kappa}_{\alpha}(\tilde{x}, \tilde{z}) \in \mathbb{R}_{\mathrm{Sym}}^{(N+1) \times(N+1)}$ is a given symmetric matrix which is positive definite.

The quantity $\tilde{v}_{\alpha}$ is the Darcy flux and $\tilde{v}_{\alpha} / \tilde{\phi}_{\alpha}$ is the velocity vector field of the fluid $\alpha$. The expression defining the flux $\tilde{v}_{\alpha}$ follows from Darcy's law (where $\tilde{\kappa}_{\alpha}=\frac{k_{\alpha}}{\mu_{\alpha}}$ with $\mu_{\alpha}$ the dynamic viscosity and $k_{\alpha}$ the intrinsic permeability tensor of the porous medium). The fourth condition of (1.2) involves the outward unit normal $\tilde{n}$ to $\tilde{\Omega}^{\tilde{t}}$ and means that the flux of freshwater can only go out of the soil (in the absence of sources), which is very natural. In our proofs, we will need the fifth condition of (1.2), which is a natural condition that means in particular that the saltwater in the soil is always below the sea level $\tilde{z}=\tilde{h}_{1}$. The sixth condition of (1.2) means that the interface $\tilde{\Gamma}_{h}^{\tilde{t}}$ is transported by the freshwater velocity vector field, the interface $\tilde{\Gamma}_{g}^{\tilde{t}}$ is transported by both the fresh and saltwater, and finally both the fresh and saltwater move tangentially to the bottom $\tilde{z}=\tilde{b}$.

We also assume the following boundary condition

$$
\left\{\begin{array}{l}
\tilde{p}(\tilde{t}, \tilde{x}, \tilde{z})= \begin{cases}\gamma_{s}\left(\tilde{h}_{1}-\tilde{z}\right) & \text { if } \quad \tilde{z}=\tilde{h}(\tilde{t}, \tilde{x})=\tilde{a}(\tilde{x})<\tilde{h}_{1} \\
0 & \text { otherwise }\end{cases} \\
\tilde{h}(\tilde{t}, \tilde{x})=\tilde{a}(\tilde{x}) \text { if } \tilde{a}(\tilde{x})<\tilde{h}_{1} .
\end{array}\right.
$$

The first condition of (1.3) follows from the fact that we assume the atmospheric pressure to be constant and normalized to zero and that the seawater is assumed to be at the hydrostatic equilibrium. We recall that the surface of the sea is assumed to be at the altitude $\tilde{h}_{1}$. When the free surface $\tilde{z}=\tilde{h}(\tilde{t}, \tilde{x})$ has no contact with the sea, then its pressure is assumed to be equal to the atmospheric pressure zero. The first and last lines of (1.3) mean that we assume the part $\tilde{a}<\tilde{h}_{1}$ to be under the seawater level.

\subsubsection{The confined aquifer}

The situation of the confined aquifer is chosen similar to the unconfined aquifer (see Figure 2). In particular we assume that $\tilde{h}_{1}>\tilde{h}$ in the neighbourhood of the points $\tilde{x} \in \mathbb{R}^{N}$ where $\tilde{h}=\tilde{a}$. This means that the freshwater only exits the porous medium to go in the seawater. The main difference is that the function $\tilde{h}(\tilde{x})$ is now a given function describing the shape of the upper confining aquifer and then is independent of time 


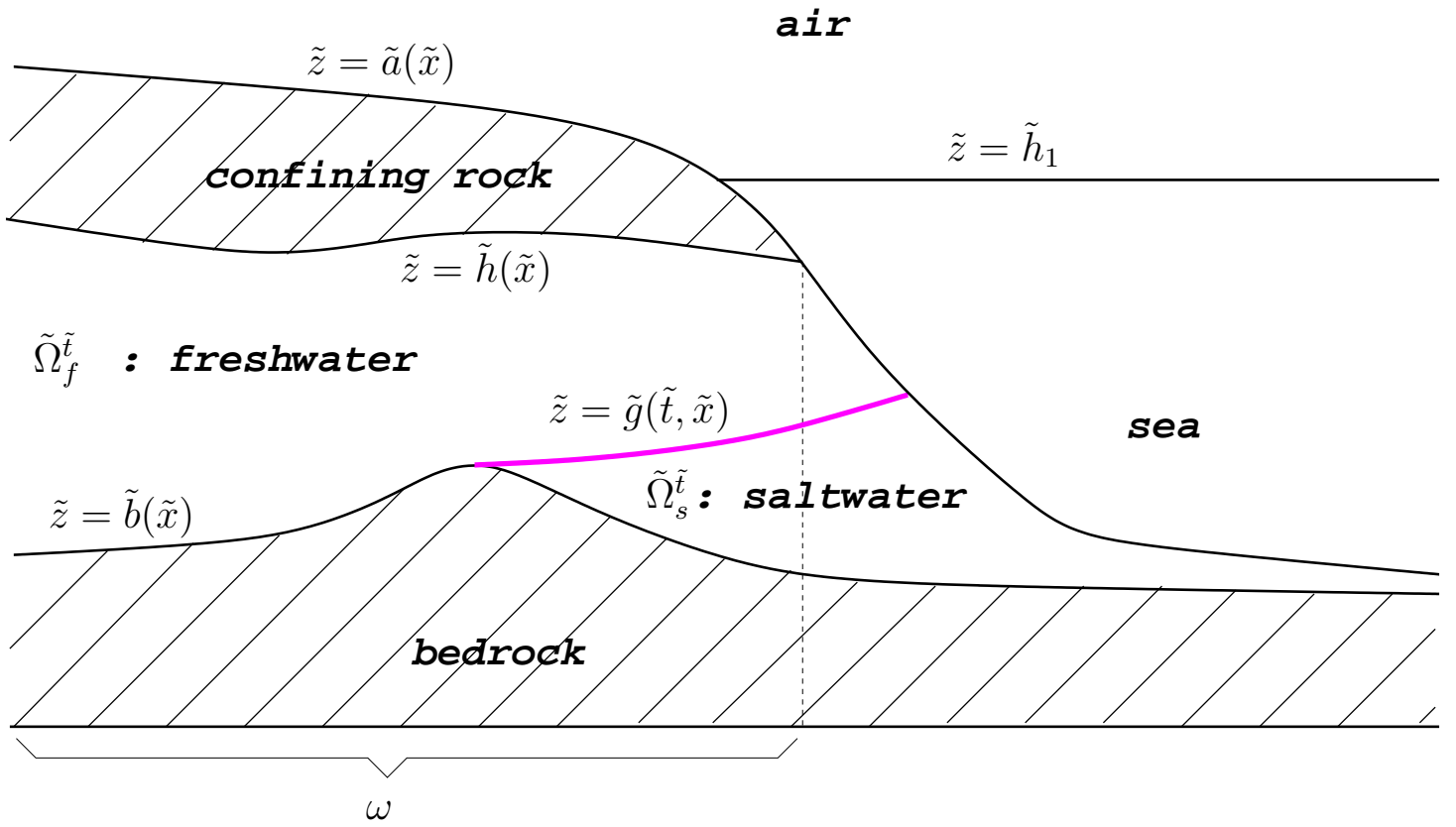

Figure 2: Confined aquifer

$\tilde{t}$. Therefore $\Gamma_{\tilde{h}}^{\tilde{t}}$ is a time independent interface. Then equations (1.2) are still satisfied, and the boundary condition (1.3) is replaced by

$$
\tilde{p}(\tilde{t}, \tilde{x}, \tilde{z})=\gamma_{s} \max \left(0, \tilde{h}_{1}-\tilde{z}\right)>0 \quad \text { for } \quad \tilde{z}=\tilde{a}(\tilde{x}) \quad \text { and } \quad \tilde{x} \in \mathbb{R}^{N} \backslash \omega
$$

with the following open, possibly unbounded set

$$
\omega=\left\{\tilde{x} \in \mathbb{R}^{N}, \quad \tilde{h}(\tilde{x})<\tilde{a}(\tilde{x})\right\},
$$

which can be seen as the horizontal projection of the region where the fluid is fully confined. Notice that in order to simplify the presentation, we assume that the pressure in (1.4) is positive, which means that the unconfined part of the soil is under the sea (like in Figure 2).

Notation. We will use the following notation. Given two functions $F$ and $G$ defined over $\mathbb{R}^{M}$, we define the set

$$
\{F<G\}:=\left\{x \in \mathbb{R}^{M}, \quad F(x)<G(x)\right\} .
$$

The same notation will be used even if one of two functions $F$ or $G$ is defined over $(0, \infty) \times \mathbb{R}^{N}$.

\subsection{Goal of the paper}

Our goal is to derive formally reduced models for the evolutions of the interfaces in the limit where the aspect ratio between the vertical dimension and the horizontal dimension is very small. We will present our precise main results in Section 2.

\subsection{Brief review of some related literature}

The literature on the subject is large, especially in hydrology. We only give here some indications on the literature, focusing more on applied mathematics.

For general models of groundwater flows, we refer the reader to $[7,6,11]$, where basically we can find two kinds of models: sharp interface models (that we consider in the present paper) and models with variable concentration of salt. For mathematical analysis, see [12] for models with variable densities or [19] for diphasic models with capillary pressure. For more historical notes on the origin of the models, see [14, 20, 27, 15].

Sharp interface models in the stationary regime have been studied mathematically, see for instance [13] for one phase problems and $[3,9,2]$ for two phase problems. 
For 2D models describing interfaces, we refer the reader to Boussinesq [10] where was derived the porous medium equation under certain assumptions. See the recent book of Vàzquez [35] for a mathematical study of this equation. Starting from sharp interface models, certain 2D models under certain assumptions are derived in the hydrological literature $[5,8,18,4]$, see also $[1,24]$ for some applications. Different models are derived in $[31,29,32]$ in the framework of variable concentration of salt.

Notice that the method we use to deduce 2D models from 3D models is similar to the one of the derivation of Saint-Venant equations from the Navier-Stokes equations (see [21]).

It is interesting to mention several works about analytical solutions and the comparison between $3 \mathrm{D}$ solutions and 2D solutions obtained after applying the Dupuit-Forchheimer approximation, see in particular $[26,36,25,22,23]$. For more information about analytical solutions, see [28, 30].

We refer to [17] for the analysis of a model similar to (2.6) in the confined case and [33] for the analysis of a stationary model similar to $(2.6),(2.8)$ (and (2.9)) in the confined case, where existence of weak solutions is proved.

Finally, for the identification of hydraulic conductivities (as an inverse problem), let us mention for instance [16].

\subsection{Organization of the paper}

In Section 2, we present our main results. In Section 3 we prove Theorem 2.2 on the identification of the limit models. In Section 4, we prove Theorem 2.3 on the oulet properties, and in Section 5, we prove Theorem 2.4 on Ghyben-Herzberg condition. Finally in Section 6 we rewrite the models under special assumptions and present some explicit particular stationary solutions.

\section{Main results}

In this section, we will explain how to obtain the reduced model (2.6) presented below, with the matrix given by (2.4). To this end, we assume the existence of a small parameter $\varepsilon>0$ such that the data of the problem satisfy

$$
\left\{\begin{array}{l}
\tilde{x}=x \\
\tilde{z}=\varepsilon z \\
\tilde{t}=t / \varepsilon \\
\tilde{a}(\tilde{x})=\varepsilon a(x) \\
\tilde{b}(\tilde{x})=\varepsilon b(x) \\
\tilde{h}_{1}=\varepsilon h_{1} \\
\tilde{\phi}_{\alpha}(\tilde{x}, \tilde{z})=\phi_{\alpha}(x, z) \\
\tilde{\kappa}_{\alpha}(\tilde{x}, \tilde{z})=\kappa_{\alpha}(x, z)=\left(\begin{array}{cc}
\kappa_{\alpha}^{x x}(x, z) & \kappa_{\alpha}^{x z}(x, z) \\
\kappa_{\alpha}^{z x}(x, z) & \kappa_{\alpha}^{z z}(x, z)
\end{array}\right) .
\end{array}\right.
$$

The parameter $\varepsilon$ can be seen as the aspect ratio between the thickness of the soil (vertical dimension) and the horizontal length of the soil. When $\varepsilon$ is small, the process is very slow and we also have to rescale the time, in order to observe some evolution of the interfaces.

In order to make precise our result (even if it is only a formal result), we need to consider the following rescaling:

$$
\left\{\begin{array}{l}
\tilde{h}(\tilde{t}, \tilde{x}) \quad=\varepsilon h^{\varepsilon}(t, x) \quad\left(\text { with } \quad h^{\varepsilon}(x) \quad \text { given in the confined case }\right) \\
\tilde{g}(\tilde{t}, \tilde{x})=\varepsilon g^{\varepsilon}(t, x) \\
\tilde{p}(\tilde{t}, \tilde{x}, \tilde{z})=\varepsilon \bar{p}^{\varepsilon}(t, x, z) \\
\tilde{v}_{\alpha}^{\tilde{x}}(\tilde{t}, \tilde{x})=\varepsilon v_{\alpha}^{x, \varepsilon}(t, x) \\
\tilde{v}_{\alpha}^{\tilde{z}}(\tilde{t}, \tilde{x})=\varepsilon^{2} v_{\alpha}^{z, \varepsilon}(t, x) .
\end{array}\right.
$$

Such a scaling may seem arbitrary at a first glance, but it comes out naturally from the formal computations, as the reader can see in the proofs. Moreover, it is very natural to think that the vertical velocity $\tilde{v}_{\alpha}^{\tilde{z}}$ is much smaller than the horizontal velocity $\tilde{v}_{\alpha}^{\tilde{x}}$, when the aspect ratio $\varepsilon$ is very small.

Indeed, the two last lines of (2.2) show that the velocity of the fluid is much more horizontal than vertical, 
which is the Dupuit-Forchheimer assumption (see for instance [34], [24]). We assume the existence of formal asymptotics:

$$
\left\{\begin{array}{l}
h^{\varepsilon}=h+\varepsilon \bar{h}_{1}+\varepsilon^{2} \bar{h}_{2}+\varepsilon^{3} \bar{h}_{3}+\ldots \\
g^{\varepsilon}=g+\varepsilon g_{1}+\varepsilon^{2} g_{2}+\varepsilon^{3} g_{3}+\ldots \\
\bar{p}^{\varepsilon}=\bar{p}+\varepsilon \bar{p}_{1}^{\varepsilon}+\varepsilon^{2} \bar{p}_{2}^{\varepsilon}+\varepsilon^{3} \bar{p}_{3}^{\varepsilon}+\ldots \\
v_{\alpha}^{x, \varepsilon}=v_{\alpha}^{x}+\varepsilon v_{\alpha, 1}^{x}+\varepsilon^{2} v_{\alpha, 2}^{x}+\varepsilon^{3} v_{\alpha, 3}^{x}+\ldots \\
v_{\alpha}^{z, \varepsilon}=v_{\alpha}^{z}+\varepsilon v_{\alpha, 1}^{z}+\varepsilon^{2} v_{\alpha, 2}^{z}+\varepsilon^{3} v_{\alpha, 3}^{z}+\ldots
\end{array}\right.
$$

Here we introduced the notation $\bar{h}_{1}, \bar{h}_{2}, \bar{h}_{3}$, etc. for the functions in the expansion in order to distinguish $\bar{h}_{1}$ from the constant $h_{1}$ defined in (2.1). Notice that this expansion is only formal and we do not intend to give a particular meaning except the one of computer algebra. In this paper, all the computations will be carried out assuming that we are allowed to make them (e.g., if the functions are smooth enough), but never checking that they have a special meaning in some particular functional space. Our goal is really to derive new models, and this is why we decided not to take care of the rigor in the sense of classical mathematical analysis.

We define

$$
\mathcal{K}_{\alpha}(x, z)=\gamma_{s} \int_{0}^{z} d \bar{z} \bar{\kappa}_{\alpha}^{x x}(x, \bar{z}) \quad \text { with } \quad \bar{\kappa}_{\alpha}^{x x}(x, z)=\kappa_{\alpha}^{x x}(x, z)-\left(\kappa_{\alpha}^{z z}(x, z)\right)^{-1} \kappa_{\alpha}^{x z}(x, z) \kappa_{\alpha}^{z x}(x, z) .
$$

We also set

$$
\Phi_{\alpha}(x, z)=\int_{0}^{z} d \bar{z} \phi_{\alpha}(x, \bar{z})
$$

and

$$
\varepsilon_{0}=\frac{\gamma_{s}-\gamma_{f}}{\gamma_{s}} \in(0,1)
$$

This quantity $\varepsilon_{0}$ is usually small (but has nothing to do with the aspect ratio $\varepsilon$ ).

\section{Unconfined aquifer}

We have for some open sets $U_{f}, U_{s}$ of $(0, \infty) \times \mathbb{R}^{N}$ (defined later)

$$
\begin{cases}b \leq g \leq h \leq a, \quad b<h \quad \text { and } \quad g \leq h_{1} & \text { on } \quad[0,+\infty) \times \mathbb{R}^{N} \\ \left(\Phi_{f}(\cdot, h)-\Phi_{f}(\cdot, g)\right)_{t}=\operatorname{div}_{x}\left(\left[\mathcal{K}_{f}(\cdot, z)\right]_{z=g}^{z=h} \nabla_{x}\left(p+\left(1-\varepsilon_{0}\right) h\right)\right) & \text { on } \quad\{h<a\} \\ \left(\Phi_{f}(\cdot, h)-\Phi_{f}(\cdot, g)\right)_{t} \leq \operatorname{div}_{x}\left(\left[\mathcal{K}_{f}(\cdot, z)\right]_{z=g}^{z=h} \nabla_{x}\left(p+\left(1-\varepsilon_{0}\right) h\right)\right) & \text { on } \quad U_{f} \\ \left(\Phi_{s}(\cdot, g)\right)_{t}=\operatorname{div}_{x}\left(\left[\mathcal{K}_{s}(\cdot, z)\right]_{z=b}^{z=g} \nabla_{x}\left(p+\left(1-\varepsilon_{0}\right) h+\varepsilon_{0} g\right)\right) & \text { on } \quad\{g<a\} \\ \left(\Phi_{s}(\cdot, g)\right)_{t} \leq \operatorname{div}_{x}\left(\left[\mathcal{K}_{s}(\cdot, z)\right]_{z=b}^{z=g} \nabla_{x}\left(p+\left(1-\varepsilon_{0}\right) h+\varepsilon_{0} g\right)\right) & \text { on } \quad U_{s},\end{cases}
$$

with

$$
\left[\mathcal{K}_{\alpha}(x, z)\right]_{z=z_{1}}^{z=z_{2}}=\mathcal{K}_{\alpha}\left(x, z_{2}\right)-\mathcal{K}_{\alpha}\left(x, z_{1}\right)
$$

and

$$
\left\{\begin{array}{l}
h=a \text { on }\left\{a<h_{1}\right\} \\
p(t, x)=p_{0}(t, x):=\left\{\begin{array}{l}
h_{1}-a(x) \text { if } h=a<h_{1} \\
0 \text { otherwise. }
\end{array}\right.
\end{array}\right.
$$

\section{Confined aquifer}

We have (2.6) with

$$
\left\{\begin{array}{l}
h_{t}=0 \\
p(t, x)=p_{0}(x):=\max \left(0, h_{1}-a(x)\right)>0 \quad \text { on } \quad\{h=a\} .
\end{array}\right.
$$


Setting

$$
\omega=\left\{x \in \mathbb{R}^{N}, \quad h(x)<a(x)\right\},
$$

then, in particular, $p$ is a solution of

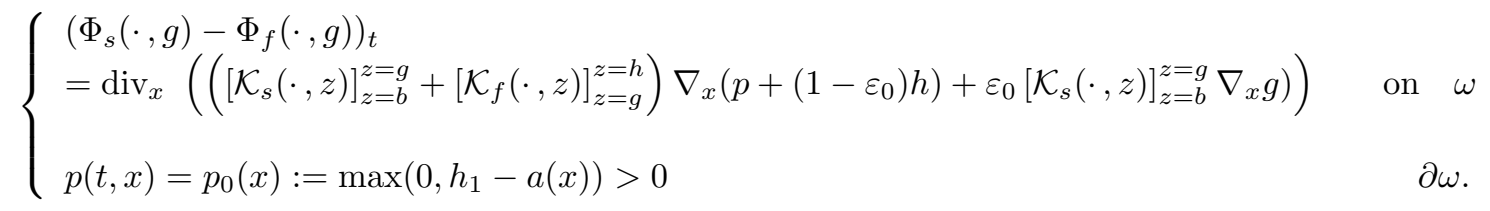

Remark 2.1 Notice that if $\phi_{f}=\phi_{s}$ (as it is expected in the physical problem), then we get zero on the left hand side of the first equation of (2.9) which becomes a stationary equation.

Then our first main result is the following formal limit:

Theorem 2.2 (The limit models)

Under the previous assumptions (in the sense of formal asymptotics), we have the following cases:

i. (unconfined case):

We assume (1.1)-(1.2)-(1.3). Then $(h, g)$ solves (2.6)-(2.7) with

$$
\left\{\begin{array}{l}
U_{f}=\{h<a\} \cup \operatorname{Int}\{g<h=a\} \cup \operatorname{Int}\{h=a=g\}, \\
U_{s}=\{g<a\} \cup \operatorname{Int}\left\{g=a<h_{1}\right\} .
\end{array}\right.
$$

ii. (confined case):

We assume (1.1)-(1.2),(1.4). Then (h,g) solves (2.6),(2.8), and $p$ solves in particular (2.9).

The coefficients $\left[\mathcal{K}_{f}(x, z)\right]_{z=g}^{z=h}$ and $\left[\mathcal{K}_{s}(x, z)\right]_{z=b}^{z=g}$ are sometimes called the transmissivity coefficients (see Section 4.4, page 140 in [6]). Notice that in the case $\mathcal{K}_{\alpha}(x, z)=z I d, \phi_{\alpha}(x, z)=1$ and $b=0$, equations (2.6) reduce to the Boussinesq equation (see equation (2) page 14 in [10]) either for $g=b$ (no saltwater) or for $h=g$ (no freshwater).

We now introduce the following additional non-degeneracy condition (which will be useful only in the confined case):

$$
-\nu^{T} \cdot\left[\mathcal{K}_{f}(x, z)\right]_{z=g}^{z=a} \cdot \nabla_{x} a>0 \quad \text { if } \quad g<a \text { for } \quad x \in \partial \omega
$$

where $\nu$ is the outward unit normal to $\omega$.

Notice that inequality $(2.10)$ holds, if $\mathcal{K}_{f}$ is proportional to the identity and if on $\partial \omega$, the vector field $\nabla_{x} a$ points inwards $\omega$. If moreover we work in dimension $N+1=2$, then condition (2.10) means that the upper boundary of the confining rock is going down into the sea (like in Figure 2). This condition is a sufficient technical condition that simplifies the analysis (in the proof of Theorem 2.3 below) in the confined case. If this condition is not be satisfied, then we cannot exclude a degenerate situation where there exists an outlet of freshwater into the seawater with non zero thickness in the limit case $\varepsilon=0$. For this reason, (2.10) is called a non-degeneracy condition.

\section{Theorem 2.3 (Outlet properties)}

We assume that $(h, g)$ are smooth enough.

i. (unconfined case): We assume that $(h, g)$ solves (2.6)-(2.7) with $U_{f}=U_{s}=[0,+\infty) \times \mathbb{R}^{N}$.

- i.1. (evolution case):

If the following condition

$$
g=a=h \quad \text { on } \quad\left\{a<h_{1}\right\}
$$

holds at time $t=0$, then it formally holds for all times, provided that it holds for all times at infinity in space in the following sense:

(2.12) there exists $R>0$ such that $g(t, x)=a(x) \quad$ if $\quad|x| \geq R \quad$ and $\quad(t, x) \in[0,+\infty) \times\left\{a<h_{1}\right\}$. 
- i.2. (stationary case):

If $h_{t}=g_{t}=0$, then we have formally (2.11) if such relation holds at infinity in space in the sense of condition (2.12).

ii. (confined case): We assume that $(h, g)$ solves (2.6),(2.8) with $U_{f}=U_{s}=[0,+\infty) \times \mathbb{R}^{N}$.

We assume moreover that the condition (2.10) is satisfied.

- ii.1. (evolution case):

If the following condition

$$
g=a \quad \text { on } \quad\{h=a\}
$$

holds at time $t=0$, then it formally holds for all times, provided that it holds for all times at infinity in space in the sense of condition (2.12).

- ii.2. (stationary case):

If $h_{t}=g_{t}=0$, then we have formally (2.13) if such relation holds at infinity in space in the sense of condition (2.12).

Theorem 2.3 shows that under suitable conditions, for the stationary limit model (2.6)-(2.7) (resp. (2.6),(2.8)), we always have $g=a=h$ on $\left\{a<h_{1}\right\}$ (resp. on $\mathbb{R}^{N} \backslash \omega$ ). This shows (at least formally) in the limit $\varepsilon \rightarrow 0$, that the region $\left\{\tilde{g}<\tilde{a}<\tilde{h}_{1}\right\}$ (resp. $\left.\{\tilde{g}<\tilde{a}\} \cap\left(\mathbb{R}^{N} \backslash \omega\right)\right)$ shrinks and disappears. The reader may have a look at Figures 1 and 2 .

We now give a result about the Ghyben-Herzberg relation (see Section 9.2 in [6]), which allows for instance in the unconfined case (see (2.14)) to compare at the equilibrium the proportions of freshwater, $h-h_{1}$ and $h_{1}-g$, respectively above the seawater level and below. By extension to the confined case, we also call (2.15) a Ghyben-Herzberg relation.

\section{Theorem 2.4 (Sufficient condition for Ghyben-Herzberg relation)}

We assume that $(h, g)$ are smooth enough.

i. (unconfined case)

If $(h, g)$ solves (2.6)-(2.7) with $U_{f}=U_{s}=[0,+\infty) \times \mathbb{R}^{N}$ and $g_{t}=h_{t}=0$, then the following Ghyben-Herzberg relation

$$
p+\left(1-\varepsilon_{0}\right) h+\varepsilon_{0} g=h_{1} \quad \text { with } \quad p=0
$$

holds on each connected component of $\{b<g<a\}:=\left\{x \in \mathbb{R}^{N}, \quad b(x)<g(t, x)<a(x)\right\}$ whose boundary intersects $\{g=a\}:=\left\{x \in \mathbb{R}^{N}, g(t, x)=a(x)\right\}$.

ii. (confined case)

If $(h, g)$ solves (2.6), (2.8) with $U_{f}=U_{s}=[0,+\infty) \times \mathbb{R}^{N}$ and $g_{t}=h_{t}=0$, then the following Ghyben-Herzberg relation

$$
p+\left(1-\varepsilon_{0}\right) h+\varepsilon_{0} g=h_{1}
$$

holds on each connected component of $\{b<g<a\}$ whose boundary intersects $\{g=a\}$.

Notice that there is no reason for the Ghyben-Herzberg condition to hold in the evolution case. Indeed $g$ would be independent of time as a consequence of the fourth line of (2.6) and $h$ would still evolve by the second line of (2.6) which could destroy the Ghyben-Herzberg relation.

\section{Identification of the limit models: proof of Theorem 2.2}

In this section we give the proof of Theorem 2.2, which is separated in two subsections: the unconfined case and the confined case. We will make some formal computations with $(h, g)$. 


\subsection{Proof in the unconfined case}

\section{Step 1: preliminaries}

Setting $\tilde{v}_{\alpha}=\left(\tilde{v}_{\alpha}^{\tilde{x}}, \tilde{v}_{\alpha}^{\tilde{z}}\right)$, we can rewrite system (1.2) as

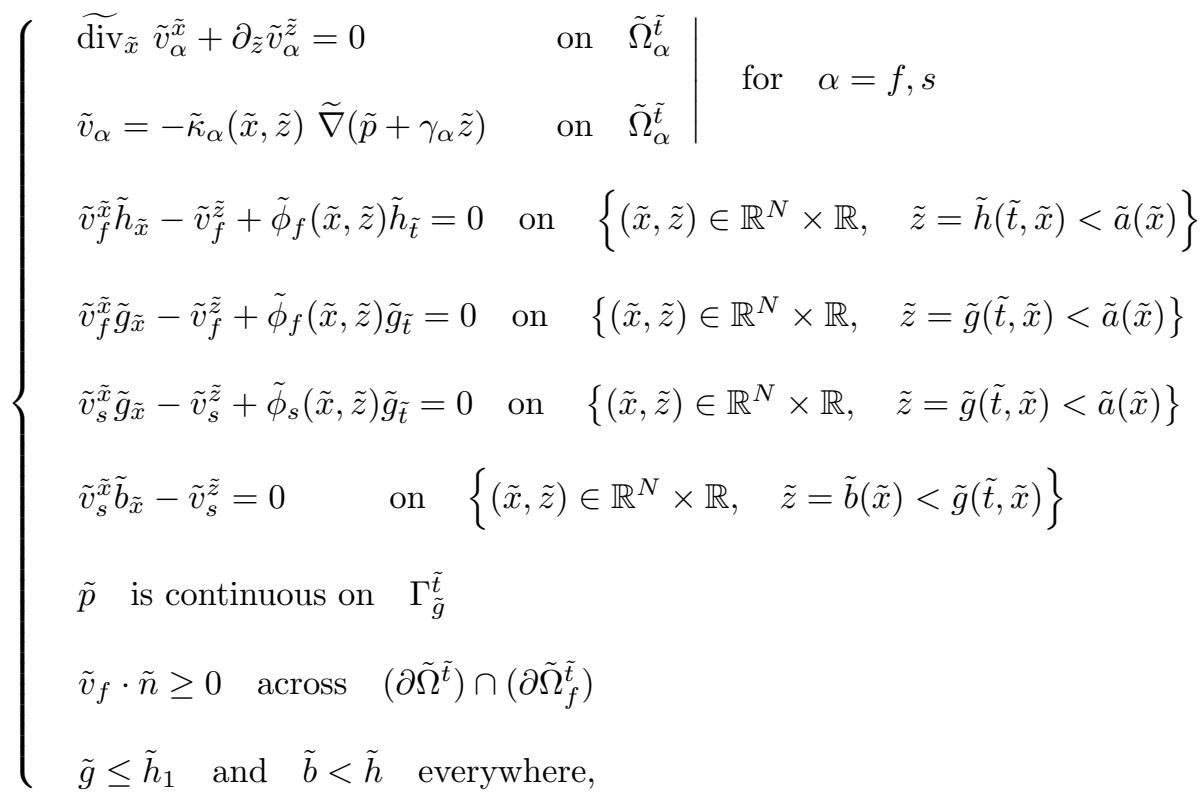

where the unit vector $\tilde{n}$ points in the same direction as $\left(\begin{array}{c}-\widetilde{\nabla}_{\tilde{x}} \tilde{a}(\tilde{x}) \\ 1\end{array}\right)=\left(\begin{array}{c}-\varepsilon \nabla_{x} a(x) \\ 1\end{array}\right)$ and

$$
\begin{cases}\tilde{p}(\tilde{t}, \tilde{x}, \tilde{z})= \begin{cases}\gamma_{s}\left(\tilde{h}_{1}-\tilde{z}\right) & \text { if } \tilde{z}=\tilde{h}(\tilde{t}, \tilde{x})=\tilde{a}(\tilde{x})<\tilde{h}_{1} \\ 0 & \text { otherwise }\end{cases} \\ \tilde{h}=\tilde{a} \quad \text { if } \quad \tilde{a}<\tilde{h}_{1} .\end{cases}
$$

\section{Step 2: rescaling}

Using the rescaling (2.1), (2.2), we set

$$
\Omega_{\alpha}^{t, \varepsilon}= \begin{cases}\left\{(x, z) \in \mathbb{R}^{N} \times \mathbb{R}, \quad g^{\varepsilon}(t, x)<z<h^{\varepsilon}(t, x)\right\} & \text { if } \quad \alpha=f, \\ \left\{(x, z) \in \mathbb{R}^{N} \times \mathbb{R}, \quad b(x)<z<g^{\varepsilon}(t, x)\right\} & \text { if } \quad \alpha=s\end{cases}
$$

and we get 


$$
\left\{\begin{array}{l}
\operatorname{div}_{x} v_{\alpha}^{x, \varepsilon}+\partial_{z} v_{\alpha}^{z, \varepsilon}=0 \quad \text { on } \quad \Omega_{\alpha}^{t, \varepsilon} \\
-\varepsilon v_{\alpha}^{x, \varepsilon}=\varepsilon \kappa_{\alpha}^{x x}(x, z) \nabla_{x} \bar{p}^{\varepsilon}+\kappa_{\alpha}^{x z}(x, z) \partial_{z}\left(\bar{p}^{\varepsilon}+\gamma_{\alpha} z\right) \quad \text { on } \quad \Omega_{\alpha}^{t, \varepsilon} \\
-\varepsilon^{2} v_{\alpha}^{z, \varepsilon}=\varepsilon \kappa_{\alpha}^{z x}(x, z) \nabla_{x} \bar{p}^{\varepsilon}+\kappa_{\alpha}^{z z}(x, z) \partial_{z}\left(\bar{p}^{\varepsilon}+\gamma_{\alpha} z\right) \quad \text { on } \quad \Omega_{\alpha}^{t, \varepsilon} \\
v_{f}^{x, \varepsilon} h_{x}^{\varepsilon}-v_{f}^{z, \varepsilon}+\phi_{f}(x, z) h_{t}^{\varepsilon}=0 \quad \text { across } \quad\left\{(x, z) \in \mathbb{R}^{N} \times \mathbb{R}, \quad z=h^{\varepsilon}(t, x)<a(x)\right\} \\
v_{f}^{x, \varepsilon} g_{x}^{\varepsilon}-v_{f}^{z, \varepsilon}+\phi_{f}(x, z) g_{t}^{\varepsilon}=0 \quad \text { across } \quad\left\{(x, z) \in \mathbb{R}^{N} \times \mathbb{R}, \quad z=g^{\varepsilon}(t, x)<a(x)\right\} \\
v_{s}^{x, \varepsilon} g_{x}^{\varepsilon}-v_{s}^{z, \varepsilon}+\phi_{s}(x, z) g_{t}^{\varepsilon}=0 \quad \text { across } \quad\left\{(x, z) \in \mathbb{R}^{N} \times \mathbb{R}, \quad z=g^{\varepsilon}(t, x)<a(x)\right\} \\
v_{s}^{x, \varepsilon} b_{x}-v_{s}^{z, \varepsilon}=0 \quad \text { across } \quad\left\{(x, z) \in \mathbb{R}^{N} \times \mathbb{R}, \quad z=b(x)<g^{\varepsilon}(t, x)\right\} \\
\bar{p}^{\varepsilon} \quad \text { is continuous on } \quad \Gamma_{g^{\varepsilon}}^{t}:=\left\{(x, z) \in \mathbb{R}^{N} \times \mathbb{R}, \quad z=g^{\varepsilon}(t, x)\right\} \\
-v_{f}^{x, \varepsilon} \cdot \nabla_{x} a+v_{f}^{z, \varepsilon} \geq 0 \quad \text { across } \quad\left\{z=h^{\varepsilon}(t, x)=a(x)>g^{\varepsilon}(t, x)\right\} \\
g \leq h_{1} \quad \text { and } b<h \quad \text { everywhere, }
\end{array}\right.
$$

with

$$
\left\{\begin{array}{l}
\bar{p}^{\varepsilon}(t, x, z)= \begin{cases}\gamma_{s}\left(h_{1}-z\right) & \text { if } z=h^{\varepsilon}(t, x)=a(x)<h_{1} \\
0 & \text { otherwise }\end{cases} \\
h^{\varepsilon}=a \quad \text { if } \quad a<h_{1} .
\end{array}\right.
$$

This implies in particular that

$$
\left\{\begin{array}{ll}
\partial_{z}\left(\bar{p}^{\varepsilon}+\gamma_{\alpha} z\right) & =O(\varepsilon)=-\varepsilon\left(\kappa_{\alpha}^{z z}(x, z)\right)^{-1}\left\{\varepsilon v_{\alpha}^{z, \varepsilon}+\kappa_{\alpha}^{z x}(x, z) \nabla_{x} \bar{p}^{\varepsilon}\right\} \\
v_{\alpha}^{x, \varepsilon}+\bar{\kappa}_{\alpha}^{x x}(x, z) \nabla_{x} \bar{p}^{\varepsilon} & =O(\varepsilon)=\varepsilon \kappa_{\alpha}^{x z}(x, z)\left(\kappa_{\alpha}^{z z}(x, z)\right)^{-1} v_{\alpha}^{z, \varepsilon}
\end{array} \mid \text { in } \Omega_{\alpha}^{t, \varepsilon},\right.
$$

with $\bar{\kappa}_{\alpha}^{x x}(x, z)$ defined in $(2.4)$.

Notice that the first line of (3.5) comes from the third line of (3.3), and the second line of (3.5) comes from inserting the first line of (3.5) into the second line of (3.3).

It is easy to check that the matrix $\bar{\kappa}_{\alpha}^{x x}(x, z) \in \mathbb{R}_{\text {sym }}^{2 \times 2}$ is symmetric positive definite because $\kappa_{\alpha}$ is symmetric positive definite.

We now set

$$
\Omega_{\alpha}^{t}= \begin{cases}\left\{(x, z) \in \mathbb{R}^{N} \times \mathbb{R}, \quad g(t, x)<z<h(t, x)\right\} & \text { if } \quad \alpha=f, \\ \left\{(x, z) \in \mathbb{R}^{N} \times \mathbb{R}, \quad b(x)<z<g(t, x)\right\} & \text { if } \quad \alpha=s .\end{cases}
$$

Using the formal asymptotics (2.3), this implies for the leading order term that:

$$
\left\{\begin{array}{l}
\partial_{z}\left(\bar{p}+\gamma_{\alpha} z\right)=0 \\
v_{\alpha}^{x}=-\bar{\kappa}_{\alpha}^{x x}(x, z) \nabla_{x} \bar{p}
\end{array} \mid \text { in } \Omega_{\alpha}^{t}\right.
$$

The second equation of (3.6) gives a kind of effective Darcy's law for the horizontal "velocity" of the fluid. The first equation of (3.6) means that the fluid is vertically at the hydrostatic equilibrium. This implies also that the velocity of the fluid is only horizontal, which is the classical formulation of the Dupuit-Forchheimer assumption (see for instance [34], [24]). 
Using again the formal asymptotics (2.3), we deduce from (3.3), the second and third lines of (3.3) being replaced respectively by the second and first lines of (3.6):

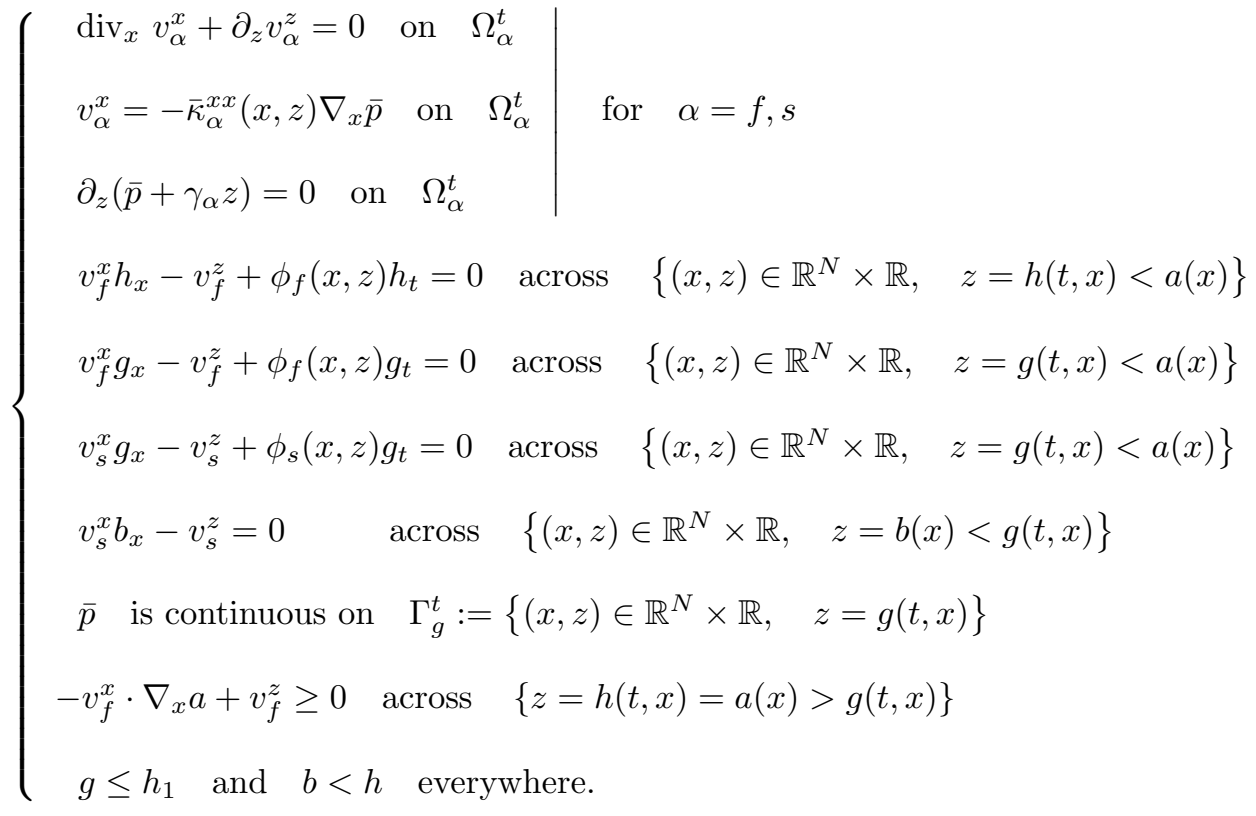

Then we can integrate the pressure in the vertical direction and get

$$
\bar{p}(t, x, z)= \begin{cases}\gamma_{s} p_{0}(t, x)+\gamma_{f}(h(t, x)-z) & \text { for } \quad g(t, x)<z<h(t, x) \\ \gamma_{s} p_{0}(t, x)+\gamma_{f}(h(t, x)-g(t, x))+\gamma_{s}(g(t, x)-z) & \text { for } \quad b(x)<z<g(t, x),\end{cases}
$$

with

$$
p_{0}(t, x):= \begin{cases}h_{1}-a(x) & \text { if } h(t, x)=a(x)<h_{1} \\ 0 & \text { otherwise }\end{cases}
$$

i.e.

$$
\gamma_{s}^{-1} \bar{p}(t, x, z)= \begin{cases}p_{0}(t, x)+\left(1-\varepsilon_{0}\right)(h(t, x)-z) & \text { for } \quad g(t, x)<z<h(t, x) \\ p_{0}(t, x)+\left(1-\varepsilon_{0}\right)(h(t, x)-g(t, x))+(g(t, x)-z) & \text { for } \quad b(x)<z<g(t, x),\end{cases}
$$

with $\varepsilon_{0}$ defined in (2.5). This shows in particular that

$$
\gamma_{s}^{-1} \nabla_{x} \bar{p}(t, \cdot)= \begin{cases}\nabla_{x}\left(p_{0}+\left(1-\varepsilon_{0}\right) h\right) & \text { on } \Omega_{f}^{t}, \\ \nabla_{x}\left(p_{0}+\left(1-\varepsilon_{0}\right) h+\varepsilon_{0} g\right) & \text { on } \Omega_{s}^{t} .\end{cases}
$$

Step 3: integration on $[g, h]$

Integrating the first equation of (3.7), we get

$$
\int_{g}^{h} d z\left(\operatorname{div}_{x} v_{f}^{x}(x, z)\right)+\left[v_{f}^{z}\right]_{z=g}^{z=h}=0
$$

and conclude that

$$
\int_{g}^{h} d z\left(\operatorname{div}_{x} v_{f}^{x}(x, z)\right)+\phi_{f}(x, h) h_{t}-\phi_{f}(x, g) g_{t}+\left(v_{f}^{x}\right)_{\mid z=h} h_{x}-\left(v_{f}^{x}\right)_{\mid z=g} g_{x}=0
$$

by the following arguments: (3.11) holds in $\{h<a\}$ by considering the fourth and the fifth lines of (3.7) respectively. 
Recall the fourth and the ninth lines of (3.7):

$$
\begin{gathered}
v_{f}^{x} h_{x}-v_{f}^{z}+\phi_{f}(x, z) h_{t}=0 \quad \text { across } \quad\{h<a\} \\
v_{f}^{x} h_{x}-v_{f}^{z} \leq 0 \quad \text { across } \quad\{h=a>g\} .
\end{gathered}
$$

We see that the outflow condition (3.13) is equivalent to assume that (3.12) still holds on $\{h=a>g\}$, but with the convention that $\left(\Phi_{f}(x, h)\right)_{t}=\phi_{f}(x, h) h_{t}$ satisfies

$$
\left(\Phi_{f}(x, h)\right)_{t} \geq 0 \quad \text { across } \quad\{h=a>g\} .
$$

With this convention of interpretation, (3.11) can be rewritten as

$$
\left(\Phi_{f}(x, h)-\Phi_{f}(x, g)\right)_{t}+\operatorname{div}_{x}\left(\int_{g}^{h} d z v_{f}^{x}(x, z)\right)=0 .
$$

Using the fact that $\nabla_{x} \bar{p}$ is independent of $z$ in $\Omega_{f}^{t}$, and setting

$$
\mathcal{K}_{\alpha}(x, z)=\gamma_{s} \int_{0}^{z} d \bar{z} \bar{\kappa}_{\alpha}^{x x}(x, \bar{z})
$$

using (3.6), we get

$$
\left(\Phi_{f}(x, h)-\Phi_{f}(x, g)\right)_{t}=\operatorname{div}_{x}\left(\left[\mathcal{K}_{f}(x, z)\right]_{z=g}^{z=h} \gamma_{s}^{-1} \nabla_{x} \bar{p}\right)
$$

i.e., using (3.10),

$$
\begin{cases}\left(\Phi_{f}(x, h)-\Phi_{f}(x, g)\right)_{t}=\operatorname{div}_{x}\left(\left[\mathcal{K}_{f}(x, z)\right]_{z=g}^{z=h} \nabla_{x}\left(p_{0}+\left(1-\varepsilon_{0}\right) h\right)\right) & \text { on } \quad\{h<a\}, \\ -\left(\Phi_{f}(x, g)\right)_{t} \leq \operatorname{div}_{x}\left(\left[\mathcal{K}_{f}(x, z)\right]_{z=g}^{z=h} \nabla_{x}\left(p_{0}+\left(1-\varepsilon_{0}\right) h\right)\right) & \text { on } \quad\{h=a>g\},\end{cases}
$$

where we have used convention (3.16) in the second line of (3.15). This implies the third line of (2.6) with

$$
U_{f}=\{h<a\} \cup \operatorname{Int}\{h=a>g\} \cup \operatorname{Int}\{h=a=g\} .
$$

Step 4: integration on $[b, g]$

We get

$$
\int_{b}^{g} d z\left(\operatorname{div}_{x} v_{s}^{x}(x, z)\right)+\left[v_{s}^{z}\right]_{z=b}^{z=g}=0
$$

i.e., as above

$$
\int_{b}^{g} d z\left(\operatorname{div}_{x} v_{s}^{x}(x, z)\right)+\phi_{s}(x, g) g_{t}+\left(v_{s}^{x}\right)_{\mid z=g} g_{x}-\left(v_{s}^{x}\right)_{\mid z=b} b_{x}=0
$$

with the following reasoning: (3.16) holds in $\{g<a\}$ by considering the sixth and the seventh lines of (3.7).

We can rewrite $(3.16)$ as

$$
\left(\Phi_{s}(x, g)\right)_{t}+\operatorname{div}_{x}\left(\int_{b}^{g} d z v_{s}^{x}(x, z)\right)=0 .
$$

Using the fact that $\nabla_{x} \bar{p}$ is independent of $z$ in $\Omega_{s}^{t}$, we get as above

$$
\left(\Phi_{s}(x, g)\right)_{t}=\operatorname{div}_{x}\left(\left[\mathcal{K}_{s}(x, z)\right]_{z=b}^{z=g} \gamma_{s}^{-1} \nabla_{x} \bar{p}\right)
$$

i.e., by the second line of (3.10)

$$
\left(\Phi_{s}(x, g)\right)_{t}=\operatorname{div}_{x}\left(\left[\mathcal{K}_{s}(x, z)\right]_{z=b}^{z=g} \nabla_{x}\left(p_{0}+\left(1-\varepsilon_{0}\right) h+\varepsilon_{0} g\right)\right) \quad \text { on } \quad\{g<a\}
$$

This implies the fifth line of (2.6) with

$$
U_{s}=\{g<a\} \cup \operatorname{Int}\left\{g=a<h_{1}\right\}
$$

because when $g=h=a<h_{1}$, definition (3.9) implies that $p_{0}+\left(1-\varepsilon_{0}\right) h+\varepsilon_{0} g$ is constant. 


\subsection{Proof in the confined case}

The procedure is exactly the same as in the unconfined case, except that $h$ is independent of the time $t$, and that the renormalized pressure $p_{0}(x)$ is replaced by

$$
p(t, x)= \begin{cases}\max \left(0, h_{1}-h(x)\right)>0 & \text { for } \quad x \in \mathbb{R}^{N} \backslash \omega \\ \text { unknown } & \text { for } \quad x \in \omega=\{h<a\} .\end{cases}
$$

On $\omega$, the pressure $p$ is unknown, but will be determined by PDE's relations below (see (3.19)).

Therefore we have

$$
\left\{\begin{array}{rlrl}
-\left(\Phi_{f}(x, g)\right)_{t} & =\operatorname{div}_{x}\left(\left[\mathcal{K}_{f}(x, z)\right]_{z=g}^{z=h} \nabla_{x}\left(p+\left(1-\varepsilon_{0}\right) h\right)\right) & \text { on } \quad & \{h<a\}=\omega \\
-\left(\Phi_{f}(x, g)\right)_{t} & \leq \operatorname{div}_{x}\left(\left[\mathcal{K}_{f}(x, z)\right]_{z=g}^{z=h} \nabla_{x}\left(p+\left(1-\varepsilon_{0}\right) h\right)\right) & \text { on } & \text { Int }\{g(t, \cdot)<h=a\} \subset \mathbb{R}^{N} \backslash \omega \\
\left(\Phi_{s}(x, g)\right)_{t} & =\operatorname{div}_{x}\left(\left[\mathcal{K}_{s}(x, z)\right]_{z=b}^{z=g} \nabla_{x}\left(p+\left(1-\varepsilon_{0}\right) h+\varepsilon_{0} g\right)\right) & \text { on } \quad & \{g(t, \cdot)<a\} \supset \omega \\
0 & \leq \operatorname{div}_{x}\left(\left[\mathcal{K}_{s}(x, z)\right]_{z=b}^{z=g} \nabla_{x}\left(p+\left(1-\varepsilon_{0}\right) h+\varepsilon_{0} g\right)\right) & \text { on } \quad \operatorname{Int}\left\{g(t, \cdot)=a<h_{1}\right\} \subset \mathbb{R}^{N} \backslash \omega .
\end{array}\right.
$$

This implies in particular that $p$ solves the following equation

$$
\begin{aligned}
& \left(\Phi_{s}(x, g)-\Phi_{f}(x, g)\right)_{t} \\
& \left.=\operatorname{div}_{x}\left(\left(\left[\mathcal{K}_{s}(x, z)\right]_{z=b}^{z=g}+\left[\mathcal{K}_{f}(x, z)\right]_{z=g}^{z=h}\right) \nabla_{x}\left(p+\left(1-\varepsilon_{0}\right) h\right)+\varepsilon_{0}\left[\mathcal{K}_{s}(x, z)\right]_{z=b}^{z=g} \nabla_{x} g\right)\right) \quad \text { on } \quad \omega .
\end{aligned}
$$

This implies the result ii. of Theorem 2.2 in the confined case.

\section{Outlet properties: proof of Theorem 2.3}

In this section, we give the proof of Theorem 2.3, which is splitted in two subsections for the unconfined and the confined case respectively.

\subsection{The unconfined case}

\section{Step 1: Proof of i.1. in the evolution case}

We assume that

$$
g(t, \cdot)=a \quad \text { on } \quad\left\{a<h_{1}\right\}, \quad \text { at } \quad t=0
$$

and want to show that this is true for all times $t \geq 0$. We have

$$
\nabla_{x}\left(p_{0}+\left(1-\varepsilon_{0}\right) h\right)=-\varepsilon_{0} \nabla_{x} a \quad \text { across } \quad\left\{h=a<h_{1}\right\},
$$

and from the third line of (2.6) and the first line of (2.7), we get

$$
-\left(\Phi_{f}(x, g)\right)_{t} \leq-\varepsilon_{0} \operatorname{div}_{x}\left(\left[\mathcal{K}_{f}(x, z)\right]_{z=g}^{z=a} \nabla_{x} a\right) \quad \text { on } \quad\left\{a<h_{1}\right\}
$$

Integrating on

$$
\omega_{h_{1}}=\left\{a<h_{1}\right\}
$$

we get that

$$
m(t):=\int_{\omega_{h_{1}}}\left(\Phi_{f}(\cdot, a)-\Phi_{f}(\cdot, g)\right) \geq 0
$$

is finite because of (2.12), and satisfies

$$
\frac{d m}{d t} \leq-\varepsilon_{0} \int_{\partial \omega_{h_{1}}} \bar{\nu}^{T} \cdot\left[\mathcal{K}_{f}(x, z)\right]_{z=g}^{z=a} \nabla_{x} a \leq 0,
$$


where the middle integral term is non-positive because $\bar{\nu}:=\frac{\nabla a}{|\nabla a|}$ is the outward normal to the set $\omega_{h_{1}}=$ $\left\{a<h_{1}\right\}$. Therefore $0 \leq m(t) \leq m(0)$ and $m(0)=0$ because of (4.1). This implies that $m(t)=0$ and by the strict monotonicity of $\Phi_{f}(x, \cdot)$ (because $\phi_{f}>0$ ), we conclude that

$$
g=a \quad \text { on } \quad \omega_{h_{1}}
$$

for all times $t \geq 0$. Moreover, we obviously have $h=g=a$ where $g=a$.

\section{Step 2: Proof of i.2. in the stationary case}

For any $z_{1}<h_{1}$, let us define the set

$$
\omega_{z_{1}}=\left\{a<z_{1}\right\} .
$$

In the case $g_{t}=0$, integrating by parts $(4.2)$ on $\omega_{z_{1}}$, we get

$$
0 \leq-\int_{\partial \omega_{z_{1}}} \bar{\nu}^{T} \cdot\left[\mathcal{K}_{f}(x, z)\right]_{z=g}^{z=a} \cdot \nabla_{x} a
$$

where $\bar{\nu}:=\frac{\nabla a}{|\nabla a|}$ is the outward normal to the set $\omega_{z_{1}}=\left\{a<z_{1}\right\}$. This implies that

$$
g=a \quad \operatorname{across} \partial \omega_{z_{1}} .
$$

Because this is true for any $z_{1}<h_{1}$, we conclude that

$$
g=a \quad \text { on } \omega_{h_{1}} .
$$

\subsection{The confined case}

\section{Step 1: proof of ii.1. in the evolution case}

We recall that

$$
-\left(\Phi_{f}(\cdot, g)\right)_{t} \leq \operatorname{div}_{x}\left(\left[\mathcal{K}_{f}(\cdot, z)\right]_{z=g}^{z=h} \nabla_{x}\left(p+\left(1-\varepsilon_{0}\right) h\right)\right) \quad \text { on } \quad[0,+\infty) \times \mathbb{R}^{N} .
$$

Integrating (by parts) this inequality on $\bar{\omega}=\mathbb{R}^{N} \backslash \omega$, we get (using $g \leq a$ ) that

$$
m(t):=\int_{\bar{\omega}}\left(\Phi_{f}(\cdot, a)-\Phi_{f}(\cdot, g)\right) \geq 0
$$

is finite because of (2.12). Moreover it satifies

$$
\frac{d m}{d t} \leq-\varepsilon_{0} \int_{\partial \bar{\omega}} \bar{\nu}^{T} \cdot\left[\mathcal{K}_{f}(x, z)\right]_{z=g}^{z=a} \nabla_{x} a \leq 0
$$

where in this subsection we define $\bar{\nu}=-\nu$ as the outward unit normal to $\bar{\omega}$, and where we have used condition (2.10). Using (2.13) at time $t=0$, we then argue as in the unconfined case (Step 1).

\section{Step 2: proof of ii.2. in the stationary case}

For any $z_{1} \in \mathbb{R}$, let us define the set

$$
\bar{\omega}_{z_{1}}=\bar{\omega} \cap\left\{a<z_{1}\right\} .
$$

In the case $g_{t}=0$, integrating by parts $(4.3)$ on $\bar{\omega}_{z_{1}}$, we get

$$
0 \leq-\int_{\partial \bar{\omega}_{z_{1}}} \bar{\nu}^{T} \cdot\left[\mathcal{K}_{f}(x, z)\right]_{z=g}^{z=a} \cdot \nabla_{x} a
$$

where we consider the new definition

$$
\bar{\nu}:= \begin{cases}-\nu & \text { if } \quad x \in \partial \bar{\omega}, \\ \frac{\nabla_{x} a}{\left|\nabla_{x} a\right|} & \text { if } \quad x \in \partial \bar{\omega}_{z_{1}} \backslash \partial \bar{\omega} .\end{cases}
$$

This implies that

$$
g=a \text { across } \partial \bar{\omega}_{z_{1}} .
$$

Because this is true for any $z_{1} \in \mathbb{R}$, we conclude that

$$
g=a \quad \text { on } \quad \bar{\omega} .
$$




\section{Proof of the Ghyben-Herzberg relation (Theorem 2.4)}

In this section we prove Theorem 2.4 .

\section{Proof of Theorem 2.4}

i) (unconfined case)

From Theorem 2.3 i.2, we have $g=a=h$ on $\left\{a<h_{1}\right\}$. Moreover we have $p=0$ on $\left\{a \geq h_{1}\right\} \supset\{g<a\}$, and in particular, we then deduce from the fourth line of (2.6) that

$$
\begin{cases}\operatorname{div}_{x}\left(\left[\mathcal{K}_{s}(x, z)\right]_{z=b}^{z=g} \nabla_{x}\left(\left(1-\varepsilon_{0}\right) h+\varepsilon_{0} g\right)\right)=0 & \text { on } D:=\{b<g<a\} \\ h=g=h_{1} & \text { across } \Gamma_{a}:=\{g=a\} \cap \partial D \\ g=b & \text { across } \Gamma_{b}:=\{g=b\} .\end{cases}
$$

The second line of (5.1) follows from the fact that

$$
\left\{a>h_{1}\right\} \subset\{g<a\}
$$

because we assume $g \leq h_{1}$ as it is written in the first line of (2.6). Indeed, recall that $D \subset\left\{a \geq h_{1}\right\}$. Therefore $\Gamma_{a} \subset\left\{a \geq h_{1}\right\}$. Moreover $\Gamma_{a} \cap\left\{a>h_{1}\right\}=\emptyset$, because of (5.2). Therefore $\Gamma_{a} \subset\left\{a=h_{1}\right\}$ and then $g=a=h_{1}=h$ on $\Gamma_{a}$, which shows the second line of (5.1).

Let

$$
\Psi:=\left(1-\varepsilon_{0}\right) h+\varepsilon_{0} g-h_{1}
$$

Using (5.1), we have

$$
\begin{cases}\operatorname{div}_{x}\left(\left[\mathcal{K}_{s}(x, z)\right]_{z=b}^{z=g} \nabla_{x} \Psi\right)=0 & \text { on } D \\ \Psi=0 & \text { across } \Gamma_{a} \\ g=b & \text { across } \Gamma_{b} .\end{cases}
$$

Multiplying the first equation in (5.3) by $\Psi$ and integrating over $D$, we get

$$
0=\int_{D} \Psi \operatorname{div}_{x}\left(\left[\mathcal{K}_{s}(x, z)\right]_{z=b}^{z=g} \nabla_{x} \Psi\right)=-\int_{D}\left(\nabla_{x} \Psi\right)^{T}\left[\mathcal{K}_{s}(x, z)\right]_{z=b}^{z=g}\left(\nabla_{x} \Psi\right) \leq 0,
$$

where the boundary terms vanish because of the two last lines of (5.3). This implies that $\nabla_{x} \Psi=0$ on $D$ and therefore $\Psi$ is constant locally. Therefore $\Psi=0$ on each connected component of $D$ whose boundary intersects $\Gamma_{a}$.

ii) (confined case)

From Theorem 2.3 ii.1, we have $g=a$ on $\{h=a\}$. From the fourth line of (2.6), we deduce in particular that

$$
\begin{cases}\operatorname{div}_{x}\left(\left[\mathcal{K}_{s}(x, z)\right]_{z=b}^{z=g} \nabla_{x}\left(p+\left(1-\varepsilon_{0}\right) h+\varepsilon_{0} g\right)\right)=0 & \text { on } D:=\{b<g<a\} \\ h=g=a & \text { across } \Gamma_{a}:=\{g=a\} \cap \partial D \\ g=b & \text { across } \Gamma_{b}:=\{g=b\} .\end{cases}
$$

Notice that the second line of (5.4) follows automatically because we have $g \leq h \leq a$. Let

$$
\Psi:=p+\left(1-\varepsilon_{0}\right) h+\varepsilon_{0} g-h_{1} .
$$

Using (5.4) and (2.8), we get again (5.3) and argue as in the unconfined case i. This completes the proof of the theorem. 


\section{Special assumptions and particular solutions}

In this section, for simplification, we assume that

$$
\mathcal{K}_{\alpha}(x, z)=z \cdot I d
$$

and

$$
\phi_{\alpha} \equiv 1
$$

We will present some explicit stationary solutions.

\subsection{Unconfined aquifer}

Any solution $(h, g)$ of the following system

$$
\begin{cases}b \leq g \leq h \leq a & \text { on } \quad[0,+\infty) \times \mathbb{R}^{N} \\ (h-g)_{t}=\operatorname{div}_{x}\left((h-g) \nabla_{x}\left(\left(1-\varepsilon_{0}\right) h\right)\right) & \text { on } \quad\{h<a\} \\ g_{t}=\operatorname{div}_{x}\left((g-b) \nabla_{x}\left(\left(1-\varepsilon_{0}\right) h+\varepsilon_{0} g\right)\right) & \text { on } \quad\{g<a\} \\ g=a=h & \text { on } \quad\left\{a \leq h_{1}\right\} \\ h<a & \text { on } \quad\left\{a>h_{1}\right\}\end{cases}
$$

is a solution of (2.6). Here the pressure $p$ has been omitted because $p=0$ if $a>h_{1}$ and $\{h<a\},\{g<a\} \subset$ $\left\{a>h_{1}\right\}$.

A particular stationary solution $(h, g)$ of $(6.2)$ satisfying moreover the Ghyben-Herzberg condition

$$
g=\max \left(b, h_{1}-\left(1-\varepsilon_{0}\right) \bar{h}\right) \quad \text { on } \quad\{g<a\}
$$

and

$$
h=\max \left(\bar{h}+b, h_{1}+\varepsilon_{0} \bar{h}\right) \quad \text { on } \quad\{h<a\}
$$

with $\bar{h}=h-g \geq 0, \bar{g}=g-b \geq 0$, is given by a solution $\bar{h}$ of the following problem

$$
\begin{cases}0=\operatorname{div}_{x}\left(\bar{h} \nabla_{x}\left\{\max \left(\bar{h}+b, h_{1}+\varepsilon_{0} \bar{h}\right)\right\}\right) & \text { on } \quad\{h<a\}=\left\{a>h_{1}\right\}, \\ \bar{h}=0 & \text { on } \quad\{h=a\}=\left\{a \leq h_{1}\right\},\end{cases}
$$

where the transition set $\bar{h}+b=h_{1}+\varepsilon_{0} \bar{h}$ can be seen as a free boundary.

\section{An example of a particular stationary solution}

We recover a classical Ghyben-Herzberg solution in a special case. Let us consider the case $b=0<h_{1}=a(0)$, $N=1$ and then $x=x_{1} \in \mathbb{R}$. We also assume that

$$
\left\{\begin{array}{l}
g(x)<h(x)<a(x) \quad \text { for } \quad x<0, \\
g(x)=h(x)=a(x) \text { for } \quad x \geq 0
\end{array}\right.
$$

We set

$$
h_{0}=\frac{h_{1}}{1-\varepsilon_{0}} .
$$

Then for any $\ell>0$, there exists an explicit solution of (6.3) given by

$$
\bar{h}(x)= \begin{cases}h_{0} \sqrt{\frac{-x}{\ell}} & \text { for } \quad-\ell \leq x<0, \\ h_{0} \sqrt{1-\varepsilon_{0}\left(\frac{x+\ell}{\ell}\right)} & \text { for } \quad x \leq-\ell .\end{cases}
$$


This corresponds to

$$
g(x)= \begin{cases}h_{1}-\left(1-\varepsilon_{0}\right) h_{0} \sqrt{\frac{-x}{\ell}} & \text { for } \quad-\ell \leq x<0 \\ 0 & \text { for } \quad x \leq-\ell,\end{cases}
$$

and

$$
h(x)= \begin{cases}h_{1}+\varepsilon_{0} h_{0} \sqrt{\frac{-x}{\ell}} & \text { for } \quad-\ell \leq x<0 \\ h_{0} \sqrt{1-\varepsilon_{0}\left(\frac{x+\ell}{\ell}\right)} & \text { for } \quad x \leq-\ell .\end{cases}
$$

This solution is displayed in Figure 3.

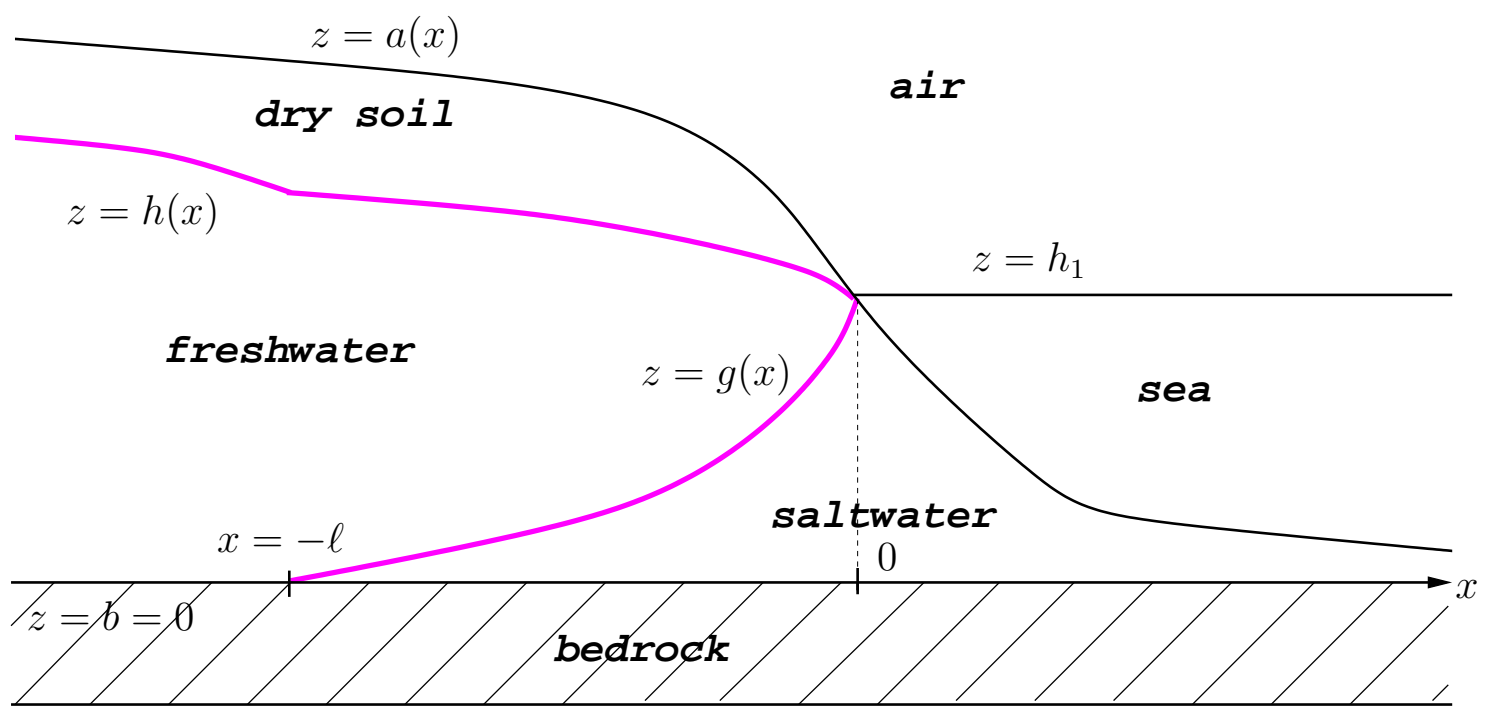

Figure 3: A classical stationary solution in the unconfined case

Remark 6.1 Notice that, because of the square root shape of the free boundary at the point $x=0$, and the infinite velocity of the freshwater at that point (because this field is divergence free), the assumption (2.2) of small and essentially horizontal velocity is no longer valid at that point. Our model seems to capture the essence of the physics, but a refined analysis would require to consider a boundary layer close to the singular points, like the outlet of freshwater into the seawater.

\subsection{Confined aquifer}

We recall that

$$
\omega=\left\{x \in \mathbb{R}^{N}, \quad h(x)<a(x)\right\} .
$$

Then $(h, g)$ is a solution of $(2.6)$ and $(2.8)$, if $(h, g)$ solves the following equations

$$
\begin{cases}b \leq g \leq h \leq a & \text { on } \quad[0,+\infty) \times \mathbb{R}^{N}, \\ g_{t}=\operatorname{div}_{x}\left((g-b) \nabla_{x}\left(p+\left(1-\varepsilon_{0}\right) h+\varepsilon_{0} g\right)\right) & \text { on } \quad \omega, \\ g=a=h & \text { on } \quad \mathbb{R}^{N} \backslash \omega,\end{cases}
$$

with $p$ solution of

$$
\begin{cases}\left.0=\operatorname{div}_{x}\left((h-b) \nabla_{x}\left(p+\left(1-\varepsilon_{0}\right) h\right)+\varepsilon_{0}(g-b) \nabla_{x} g\right)\right) & \text { on } \quad \omega, \\ p(t, x)=p_{1}(x):=\max \left(0, h_{1}-a(x)\right)>0 & \text { across } \quad \partial \omega .\end{cases}
$$




\section{Proposition 6.2 (horizontal confinement)}

We assume that $h \equiv h_{0} \in\left(0, h_{1}\right)$ on $\omega$ and $b \equiv 0$. Then the solution $g$ of (6.4)-(6.5) satisfies for all times $t>0$

$$
\begin{cases}0 \leq g \leq h_{0} & \text { on } \omega, \\ g=h_{0} & \text { across } \partial \omega, \\ g_{t}=\varepsilon_{0} \operatorname{div}_{x}\left(g\left(1-\frac{g}{h_{0}}\right) \nabla_{x} g\right)+\varepsilon_{0} \operatorname{div}_{x}\left(g \nabla_{x} \beta\right) & \text { on } \omega,\end{cases}
$$

with $\beta$ solution of

$$
\left\{\begin{array}{l}
\Delta \beta=0 \quad \text { on } \omega, \\
\beta=\frac{h_{0}}{2} \quad \text { across } \quad \partial \omega .
\end{array}\right.
$$

Remark 6.3 The third equation of (6.6) appears to be an approximation of the equation considered in [34], in the limit case where the gradient of the solution is small.

\section{Proof of Proposition 6.2}

We simply set

$$
\beta(t, x)=\varepsilon_{0}^{-1}\left(p(t, x)-\left(h_{1}-h_{0}\right)\right)+\frac{1}{2 h_{0}} g^{2}(t, x) .
$$

The end of the proof is straightforward.

Remark 6.4 Because $h-b$ is constant under the confining rock (in $\omega$ ), notice that

$$
(h-b) \nabla_{x}\left(p+\left(1-\varepsilon_{0}\right) h\right)+\varepsilon_{0}(g-h) \nabla_{x} g
$$

is then proportional to $\nabla_{x} w$. In dimension $N=1$, we have $\Delta w=\nabla_{x}\left(\nabla_{x} w\right)=0$ in $\omega$, which means that $\nabla_{x} w$ is constant and can then be assumed to be a given quantity (proportional to the velocity of freshwater arriving from the left in Figure 4). In higher dimensions, we can solve uniquely equation (6.7), if we have further suitable boundary conditions far from $\partial \omega$ or at infinity.

\subsubsection{A stationary solution}

Proposition 6.5 Under assumptions of Proposition 6.2, we consider a solution $w$ of

$$
\left\{\begin{array}{l}
\Delta w=0 \quad \text { on } \omega, \\
w=\frac{h_{0}}{2} \quad \text { across } \quad \partial \omega,
\end{array}\right.
$$

and we assume the following Ghyben-Herzberg relation

$$
g-\frac{g^{2}}{2 h_{0}}+w=h_{0} \quad \text { on } \quad\{g>0\} .
$$

Then any solution of (6.6)-(6.7)-(6.8) satisfies

$$
\left\{\begin{array}{l}
g_{t}=0 \\
\{g>0\}=\left\{w<h_{0}\right\} .
\end{array}\right.
$$

The proof of Proposition 6.5 is straightforward.

\section{An example of a particular stationary solution}

We will give a solution in dimension 1 . Let us consider the case $b=0<h_{0}=a(0), N=1$ and then $x=x_{1} \in \mathbb{R}$. We assume that

$$
\omega=\{x<0\} .
$$


Under the assumptions of Proposition 6.5, for any $\ell>0$, there exists an explicit solution given by

$$
w(x)=\frac{h_{0}}{2}\left(1-\frac{x}{\ell}\right)
$$

and

$$
\begin{cases}g-\frac{g^{2}}{2 h_{0}}+w=h_{0} & \text { for } \quad-\ell<x<0 \\ g=0 & \text { for } \quad x \leq-\ell .\end{cases}
$$

This solution is displayed in Figure 4.

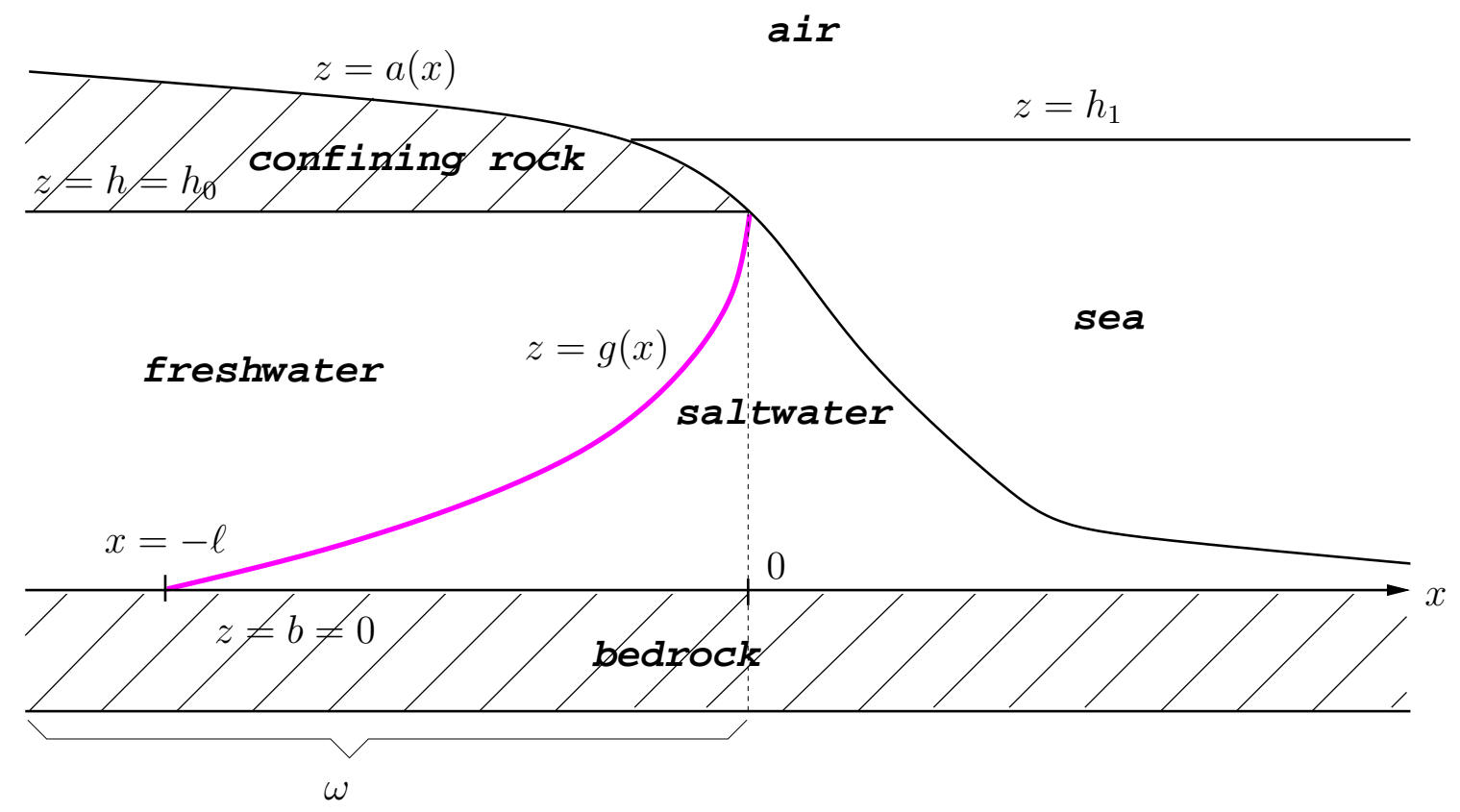

Figure 4: A stationary solution in the confined case

\section{Acknowledgments}

The authors would like to thank A. Al Bitar and R. Ababou for enlighting discussions and for indications to the literature on the subject. A discussion with R. Ababou was useful to correct a mistake in a previous version of the manuscript. The authors also would like to thank A. Fino for helpful discussions, and G. Chmaycem, S. Issa and H. Moustafa for useful remarks on the manuscript. The authors finally thank the two unknown referees for their careful reading of the manuscript, their suggestions and numerous corrections that helped to improve substantially the presentation of the paper.

\section{References}

[1] A. Al Bitar, Modélisation des écoulements en milieu poreux hétérogènes 2D / 3D, avec couplages surface / souterrain et densitaires, Thèse de l'Institut National Polytechnique de Toulouse, (2007).

[2] G. Alduncin, J. Esquivel-Avila, L. Reyes-Avila, Steady filtration problems with seawater intrusion: variational analysis, Symposium on Advances in Computational Mechanics, Vol. 3 (Austin, TX, 1997). Comput. Methods Appl. Mech. Engrg. 151 (1-2) (1998), 13-25.

[3] H.W. Alt, C.J. van DuiJn, A stationary flow of fresh and salt groundwater in a coastal aquifer, Nonlinear Analysis, Theory, Methods \& Applications 14 (8) (1990), 625-656.

[4] M. BAKKeR, Simple groundwater flow models for seawater intrusion, In: Proceedings of SWIM16, Wolin Island, Poland, (2000). 
[5] J. Bear, Hydraulics of Groundwater, McGrraw-Hill, New York, (1979).

[6] J. Bear, A.H.D. Cheng, Modeling Groundwater Flow and Contaminant Transport, Theory and applications of transport in porous media 23, Springer (2010).

[7] J. Bear, A.H.D. Cheng, S. Sorek, D. Ouazar, I. Herrera (Eds.), Seawater Intrusion in Coastal Aquifers - Concepts, Methods and Practices, Theory and applications of transport in porous media 14, Kluwer Academic Publisher, Netherlands, (1999).

[8] J. Bear, A. VerruiJt, Modelling groundwater flow and pollution, D. Reidel Publishing Company, Dordecht, Holland, (1987).

[9] A. Bonnet, R. Monneau, On the Mushy Region Arising Between Two Fluids in a Porous Medium, Nonlinear Analysis, Real World and Applications 5 (1) (2004), 159-182.

[10] J. BoussinesQ, Recherches théoriques sur l'écoulement des nappes d'eau infiltrées dans le sol et sur le débit des sources, Journal de mathématiques pures et appliquées 5ème série, tome 10 (1904), 5-78.

[11] W.C. Burnett, P.K. Aggarwal, A. Aureli, H. Bokuniewicz, J.E. Cable, M.A. Charette, E. Kontar, S. Krupa, K.M. Kulkarni, A. Loveless, W.S. Moore, J.A. Oberdorfer, J. Oliveira, N. Ozyurt, P. Povinec, 1, A.M.G. Privitera, R. Rajar, R.T. Ramessur, J. Scholten, T. Stieglitz, M. Taniguchi, J.V. Turner, Review: Quantifying submarine groundwater discharge in the coastal zone via multiple methods, Science of The Total Environment 367 (2-3) (2006), 498-543.

[12] Z. Chen, R.E. Ewing, Mathematical analysis for reservoir models, SIAM J. Math. Anal. 30 (1999), 431-453.

[13] M. Снipot, Variational inequalities and flow in porous media, Applied Mathematical Sciences, 52. New York etc.: Springer-Verlag, (1984).

[14] H. DARCy, Les fontaines publiques de la ville de Dijon; exposition et application des principes à employer dans les questions de distribution d'eau. Victor Dalmont, Editeur, Paris, (1856).

[15] J. Dupuit, Etudes théoriques et pratiques sur le mouvement des eaux dans les canaux découverts et à travers les terrains perméables, 2ème édition, Dunod, Paris, (1863).

[16] M. El Alaoui Talibi, D. Ouazar, M.H. Tber, Identification of the hydraulic conductivities in a saltwater intrusion problem, J. Inverse Ill-Posed Probl. 15 (9) (2007), 935-954.

[17] M. El Alaoui Talibi, M.H. Tber, Existence of solutions for a degenerate seawater intrusion problem, Electron. J. Differential Equations 72 (2005), 1-14.

[18] H.I. Essaid, A multilayered sharp interface model of coupled freshwater and saltwater flow in a coastal system: Model development and application, Water Ressources Research 26 (7) (1990), 14311454 .

[19] R. Eymard, R. Herbin, A. Michel, Mathematical study of a petroleum-engineering scheme, ESAIM: Mathematical Modelling and Numerical Analysis 37 (6) (2003), 937-972.

[20] C.W. Fetter JR, Hydrogeology: A Short History, Part 2, Ground Water, 42 (2004), 949-953.

[21] J.-F. Gerbeau, B. Perthame, Derivation of viscous Saint-Venant system for laminar shallow water; numerical validation, Discrete and Continuous Dynamical Systems - Series B 1 (1) (2001), $89-102$.

[22] A. R. Kacimov, Yu. V. Obnosov, Analytical solution for a sharp interface problem in sea water intrusion into a coastal aquifer, Proc. R. Soc. Lond. A 457 (2001), 3023-3038.

[23] A. R. Kacimov, Yu. V. Obnosov, M. M. Sherif and J. S. Perret, Analytical Solution to a Sea-water Intrusion Problem with a Fresh Water Zone Tapering to a Triple Point, Journal of Engineering Mathematics 54 (3) (2006), 197-210. 
[24] C. KAO, Fonctionnement hydraulique des nappes superficielles de fonds de vallées en interaction avec le réseau hydrographique, Thèse de doctorat en Sciences de l'Eau, Ecole Nationale du Génie Rural, des Eaux et Forêts (Paris), (2002).

[25] G. KeAdy, The Dupuit Approximation for the Rectangular Dam Problem, IMA Journal of Applied Mathematics 44 (1990), 243-260.

[26] M. Kemblowski, The Impact of the Dupuit-Forchheimer Approximation on Salt-Water Intrusion Simulation, Ground Water 25 (3) (1987), 331-336.

[27] C.M. Marle, Henry Darcy et les écoulements de fluides en milieu poreux, Oil \& Gas Science and Technology Rev. IFP, Vol. 61 (5) (2006), 599-609.

[28] P.Y. Polubarinova-Kochina, Theory of Groundwater Movement, translated from Russian edition 1952 by R.J.M. De Wiest. Princeton University Press, New Jersey, (1962).

[29] S. Sorek, V. Borisov, A. Yakirevich, A Two-Dimensional Areal Model for Density Dependent Flow Regime, Transport in Porous Media 43 (2001), 87-105.

[30] O.D.L. Strack, Groundwater Mechanics, Prentice Hall, Englewood Cliffs, NJ, (1989).

[31] O.D.L. Strack, A Dupuit-Forchheimer model for three-dimensional flow with variable density, Water Resources Research 31 (12) (1995), 3007-3017.

[32] O.D.L. Strack, R.J. Barnes, A. VerruiJt, Vertically Integrated Flows, Discharge Potential, and the Dupuit-Forchheimer Approximation, Special Issue: Ground Water Flow Modeling with the Analytic Element Method, Ground Water 44 (1) (2006), 72-75.

[33] M.H. TBER, On a seawater intrusion problem, Appl. Sci. 9 (2007), 163-173.

[34] C.J. van Duyn, D. Hilhorst, On a doubly nonlinear diffusion equation in hydrology, Nonlinear analysis, Theory, Methods and applications 11 (3) (1987), 305-333.

[35] J.L. VAzQuez, The porous medium equation: mathematical theory, Oxford University Press, USA, (2007).

[36] E.G. Youngs, An examination of computed steady-state water-table heights in unconfined aquifers: Dupuit-Forchheimer estimates and exact analytical results, J. Hydrol. 119 (1990), 201-214. 